\section{Fortbildungskongress, Davos 2005}

\section{Neurodermitis juckt anders}

\author{
Patienten mit atopischer Dermatitis, Urtikaria oder Prurigo simplex \\ subacuta leiden stark unter ihrem Juckreiz. Doch Pruritus ist nicht \\ gleich Pruritus. Patienten mit Neurodermitis weisen eine ganz \\ eigene Verarbeitung im Zentralnervensystem auf.
}

D er Einsatz einer visuellen Analogskala gebe nur ungenügend Aufschluss über die Qualität von Juckreiz, betonte Priv.-Doz. Dr. Ulf Darsow aus München. So ist der mit diesem Hilfsmittel gemessene Juckreiz bei atopischem Ekzem, Urtikaria oder Prurigo simplex subacuta nur scheinbar vergleichbar. Der Eppendorfer Juckreizfragebogen gibt dagegen ein differenziertes quantitatives und qualitatives Bild mit Hinweisen sowohl auf zentralnervöse als auch periphere Aspekte. Gerade die affektiven Begriffe des Fragebogens zeigen die Unterschiede zwischen atopischer Dermatitis und Urtikaria auf. So bezeichnen Neurodermitiker im Vergleich zu Urtikariapatienten ihren Juckreiz viel häufiger als marternd, zermürbend, ekelhaft, unbeherrschbar, unkontrollierbar oder zwanghaft (Abb.).

\section{Immer nur Juckreiz und kein Schmerz}

Bei Patienten mit atopischer Dermatitis scheint die zentralnervöse Verarbeitung eine Veränderung der Juckreizwahrnehmung auszulösen. Es gilt nicht wie bei anderen Pruritus auslösenden Faktoren, dass beim Aufkratzen der quälende Juckreiz durch $S c h m e r z$ verdrängt wird. Das zeigte eine japanische Studie mit $25 \mathrm{Neu}-$ rodermitis-Patienten, neun Psoriasis-Patienten und 20 hautgesunden Kontrollen. Die Probanden wurden standardisiert mechanisch, elektrisch, thermisch oder chemisch durch einen niedrigen $\mathrm{pH}$ Wert stimuliert. Die Psoriatiker wie auch die Kontrollen gaben als Reaktion Schmerz an, die Patienten mit atopischer Dermatitis dagegen Juckreiz. Möglicherweise sensibilisiert die chronische prurizeptive Belastung zentrale Verarbeitungsinstanzen, die nozizeptive Afferenz kann den Juckreiz nicht mehr hemmen, son- dern wird selbst als Pruritus wahrgenommen.

Stimuliert man hautgesunde Probanden am Arm, so zeigt sich in der Positronen-Emissions-Tomografie des Gehirns eine Aktivität in verschiedenen Kortexarealen. Neben sensorischen sind auch motorische Areale und solche zur Handlungsplanung beteiligt, was die die direkte Verbindung zwischen Juckreiz und Kratzen unterstreicht. Auch sind Areale beteiligt, die an der Bewusstwerdung von Reizen ebenso Anteil haben wie an der Verschaltung auf das vegetative Nervensystem, z. B. die Insula der linken Hemisphäre, die an der sensorischen Beurteilung von Reizen beteiligt ist, oder der Gyrus frontalis medialis als Areal, das für Motivation, Planung und Kognition wichtig ist. Wenn die Bedeutung der zentralnervösen Verarbeitung so groß ist, könnten auch zentralnervöse Therapieansätze wirken.

\section{Besserung durch Akupunktur}

Die Münchner Arbeitsgruppe um Darsow evaluierte vor diesem Hintergrund an gesunden Probanden die Wirksamkeit der Akupunktur bei einem Histamin-induzierten, schmerzfreien Juckreiz. Die prospektive randomisierte kontrollierte Crossover-Studie mit zehn Probanden hatte zum Ziel, Juckreizintensität, -qualität und Hautreaktionen (Quaddel, Erythem) mit und ohne Akupunktur zu vergleichen. Als Verum-Akupunkturpunkt wurde der Quchi-Punkt (Di 11) gewählt, ein Punkt im M. deltoideus diente als Plazebopunkt. Die Nadelinsertion erfolgte 1,5-2,5 cm tief mit einer Dauer von 15 Sekunden. Tatsächlich konnte die Verumakupunktur die Juckreizintensität, gemessen mit der visuellen Analogskala deutlich gegenüber einer Kontrolle ohne Akupunktur, aber auch gegenüber der Plazeboakupunktur senken. Die qualitative Erfassung der Juckreizparameter zeigte eine Reduktion besonders bei emotionalen Begriffen durch die Akupunktur. Da diese affektiven Aspekte so typisch für Neurodermitis sind, könnte Akupunktur möglicherweise zur Linderung dieses Pruritus eingesetzt werden.

\section{Darsow U. Juckreiz: Wahrnehmung und Modulation. 21. Fortbildungskongress „Fortschritte der Allergologie, Immunolo- gie und Dermatologie“, Davos, 7.-10. Sep- tember 2005}

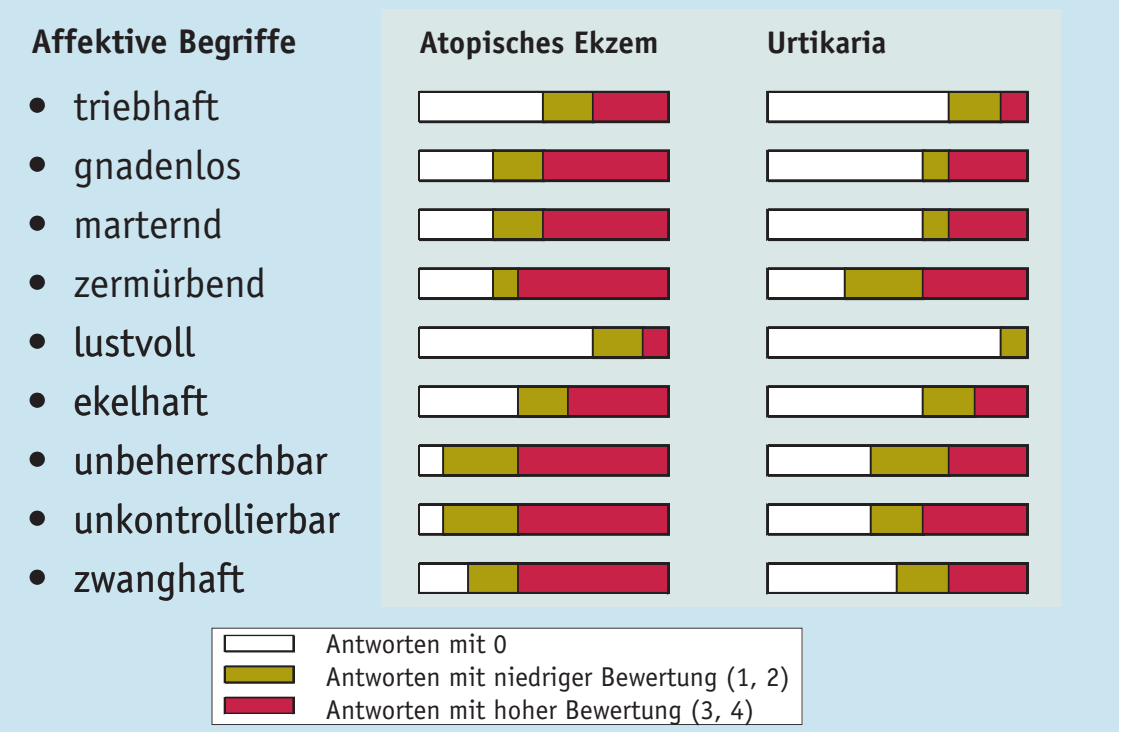

Die Qualität des Juckreizes, abgefragt mit verschiedenen affektiven Begriffen, beim atopischen Ekzem unterscheidet sich signifikant von derjenigen bei Urtikaria $(p<0,05)$. 